\title{
Values Impacting the Design of an Adaptive Educational Storybook
}

\author{
Jason B. Alonso, Angela Chang, and Cynthia Breazeal \\ MIT Media Lab \\ 20 Ames St, Cambridge, MA 02139 \\ \{jalonso, anjchang, cynthiab\}@media.mit.edu
}

\begin{abstract}
We present an adaptive educational narrative that combines an interactive storybook with drama management. By understanding our underlying value system, we are able to create an adaptive narrative that provides features adequate for the target context. Our value system and design needs invite a reexamination and reapplication of interactive storytelling systems, and results in an adaptive narrative framework that is both feasible and extensible to a wide range of experiences.
\end{abstract}

Keywords: Interactive narrative, drama management, design values, early childhood education, design process.

\section{Introduction}

Storytelling, especially in early childhood, combines learning techniques with fantasy play and artistic expression. In emergent literacy, when children are just learning to read for the first time, parents are the first educators, guiding their children through shared reading behaviors to introduce them to story, art, and print 3. Parents are observant guides, providing responsive and intelligent encouragement and demonstration in the learning process for their children. We have designed and implemented storytelling software to assist in this learning process, as an assistant that unobtrusively prompts the readers to engage in storytelling practice [1. The criticality of learning at this young age however, compels us to examine our presentation of material to children [4] - in our case, this is manifest in the nature and choice of interactive narrative framework.

In this paper, we lay out a vision for a learning tool and our values (informed by principles in early childhood education) that guide our design choices.

\section{Vision}

We envision interactive storybooks that grow as their authors continue to expand their materials (cloud storytelling) at the same time that they learn from their usage patterns to improve their performance for individuals (adaptive storytelling). Our vision is inspired from fiction, especially the illustrated primer from Stephenson's The Diamond Age [5], yet it builds upon prior work. 


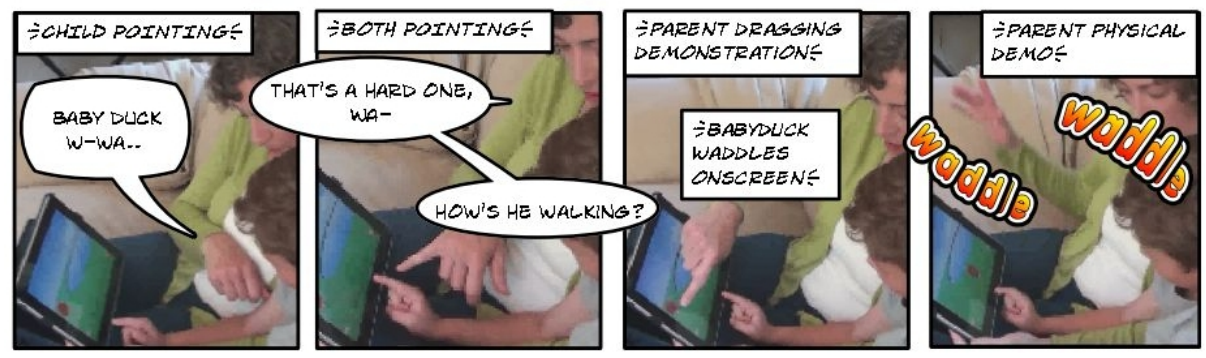

Fig. 1. Parent-child discussions prompted by TinkRBook

Cloud TinkRBook is a project building upon TinkRBook [2] and Dramamatic, an interactive narrative engine we are building for use in Cloud TinkRBook and other projects. A TinkRBook is an interactive storybook that allows users to explore how text changes in response to user interactions with story elements within a story context. The system is designed to promote parent-child discussion, as in Figure 1

We envision a system that draws its resources from the cloud (that is, its authors, who may be many, continually expand its body of story resources) with the intention that it has an ever growing body of resource from which to tell stories. As such, it will need an intelligent system to help each person find the story that is relevant to them. As this intelligence is fundamentally an interactive narrative system, Cloud TinkRBook is an adaptive educational narrative that combines an interactive storybook with drama management.

\section{$3 \quad$ Values}

By explicitly articulating the values for our design, we constrain the problem of designing an adaptive educational narrative 1 . Obviously, designing explicitly for children requires awareness of the cognitive abilities of young and beginning readers. Other considerations arise from empathizing with the other stakeholders in developing the experience, such as parents and story creators. Parents are usually the mediators for the book, and we are interested in providing some benefit to them for reading an adaptive narrative to their child. Story creators, who may be educators, artists, or authors, need to have a way to express their ideas to accomplish their educational and experiential goals.

We use the aesthetic values summarized in Table 1 to guide our design of an interactive storytelling system (outlined in the same table).

\section{Discussion}

Our design criteria, including our vision for Cloud TinkRBook and our values, present interesting challenges for interactive narrative systems and their

\footnotetext{
${ }^{1}$ Our decision to articulate values stems from a movement in the Interaction Design
} and Children (IDC) community to make values underlying design explicit 6]. 
Table 1. Impact of values on design choices

Value Design Choice

$\begin{array}{ll}\text { Leveraging } & - \text { use of explicit page turning buttons } \\ \text { existing ritual } & - \text { use of tablet form to promote physical proximity } \\ & - \text { semantic highlighting in response to strumming of words }\end{array}$

Developmental Appropriateness

- avoiding text in menus and complex HCI interaction paradigms (e.g. checkboxes, sliders, double-click)

- concise and simple text

- choice (decision points) are explicitly offered through narrative text layout or interactive graphic elements

- multisensory interaction: every touch on an active story element responds with sound or animation

- modular scene-based story structure

- small world containing educational objective within each scene

- immediately observable (short) causal links in the narrative

Addressing the Dual Audience
- providing demonstrable swapping of words to demonstrate narrative flexibility

- customization of narrative and story presentation over repeat readings

interfaces. In particular, we needed our educational concepts to prevail over interactive narrative structure, and existing systems did not fit our value system. In our review of systems, which we do not present in this paper, we found our work has a few notable differences from other systems.

Interactive storybooks may treat time as an explicit decision. How do we represent time? What does "going back" mean? In TinkRBook, when the user goes back a page, they are not going back in time as they would with a paper storybook. The state of the world and character remains consistently in the now, with the pages providing spatial distance between scenes. Choices made in a frame are generally reversible, meaning that the user can usually change a decision by revisiting the appropriate scene, and the implications of the change will propagate to other frames. For example, a child can revisit a frame where he or she can change a character's color, altering the character's presentation in all other frames.

Causal chains do not have to be long. In many interactive narrative systems, having large causal chains or complex puzzles can be enjoyable. Because we have chosen to use modular self-contained scenes, the overall narrative experience can distract from reading a story. Creating cohesion among different story scenes is an interesting challenge. Our answer so far is to use small chains of causality, such as making most choices immediately demonstrable within a scene and at 
most looping over only two frames. In the Babdyduck story for TinkRBook, instead of letting the protagonist advance to a bed time scene while covered in mud, the protagonist is sent back to a scene with a pond and a mud puddle to bathe.

The narrative experience can adapt to the user's development. We intend to keep beginnings and endings consistent and mostly change the content in the middle. Having different educational lessons sandwiched within a story framework can be enjoyable and useful for pedagogical purposes, as children learn to care for a reoccurring character and become familiar with the story world. However, too much variation between each scene can be narratively distracting.

An interactive storybook that creates a cohesive storytelling experience for a dual audience will need to adapt the narrative over a history of readings, and seek ways to foster fresh dialogue between people of different abilities. This brings up interesting idea of long term interaction with interactive narratives. How can the narrative experience adapt to the user's development?

The state of the story changes over successive readings. We have to explicitly decide which aspects of the world are persistent from the last time it was read. Our system must judge how many times users have to read a particular sequence before changing the narrative structure. For example, we can save the color choice for a protagonist and have it persistent each time the story is read. Over time the color selection scene may disappear if children don't use that scene.

\section{Summary}

In this paper, we presented an articulated need for adaptive narratives in the domain of educational storytelling. From there, we showed our vision for an interactive educational storybook along with a value-based approach to designing an interactive storytelling system that could realize our vision. In this process, we questioned basic assumptions, like how time should flow in a storybook.

\section{References}

1. Chang, A.: TinkRBooks: Tinkerable Story Elements for Emergent Literacy. Ph.D. thesis, Massachusetts Institute of Technology (2011)

2. Chang, A., Breazeal, C.: TinkRBook: shared reading interfaces for storytelling. In: Proceedings of the 10th International Conference on Interaction Design and Children (IDC 2011), pp. 145-148. ACM, New York (2011)

3. Honig, A.S., Brophy-Her, H.E.: Talking with Your Baby: Family As the First School. Syracuse University Press, Syracuse (1996)

4. Nikolajeva, M., Scott, C.: How Picturebooks Work. Psychology Press (2001)

5. Stephenson, N.: The Diamond Age, or, A Young Lady's Illustrated Primer. Bantom Books, New York (1995)

6. Yarosh, S., Radu, I., Hunter, S., Rosenbaum, E.: Examining values: an analysis of nine years of IDC research. In: Proceedings of the 10th International Conference on Interaction Design and Children (IDC 2011), pp. 136-144. ACM, New York (2011) 